\section{(6) OPEN ACCESS}

\title{
Cereblon modulator iberdomide induces degradation of the transcription factors Ikaros and Aiolos: immunomodulation in healthy volunteers and relevance to systemic lupus erythematosus
}

\author{
Peter H Schafer, ${ }_{1}^{1}$ Ying Ye, ${ }^{2}$ Lei Wu ${ }^{1}$ Jolanta Kosek, ${ }^{1}$ Garth Ringheim, ${ }_{1}$ Zhihong Yang, ${ }^{3}$ \\ Liangang Liu, ${ }^{3}$ Michael Thomas, ${ }^{2}$ Maria Palmisano, ${ }^{2}$ Rajesh Chopra ${ }^{1,4}$
}

\section{Handling editor Josef $S$ Smolen \\ - Additional material is published online only. To view please visit the journal online (http://dx.doi.org/10.1136/ annrheumdis-2017-212916) \\ 'Department of Translational Development, Celgene Corporation, Summit, New Jersey, USA \\ ${ }^{2}$ Department of Clinical Pharmacology, Celgene Corporation, Summit, New Jersey, USA \\ ${ }^{3}$ Department of Biostatistics, Celgene Corporation, Summit, New Jersey, USA \\ ${ }^{4}$ Division of Cancer Therapeutics, The Institute of Cancer Research, London, UK}

Correspondence to Dr Peter H Schafer, Department of Translational Development, Celgene Corporation, Summit, NJ 07901, USA

pschafer@celgene.com

This manuscript is based on work previously presented and published as a conference abstract at (a) The American College of Rheumatology Annual Scientific Meeting, San Diego, USA, 26-30 October 2013, and (b) The European League Against Rheumatism Annual European Congress of Rheumatology, Rome, Italy, 10 13 June 2015.

Received 28 December 2017 Revised 31 May 2018 Accepted 11 June 2018 Published Online First 26 June 2018

Check for updates

To cite: Schafer $\mathrm{PH}, \mathrm{Ye} \mathrm{Y}_{\text {, }}$ Wu L, et al. Ann Rheum Dis 2018:77:1516-1523.

\section{ABSTRACT}

Objectives IKZF1 and IKZF3 (encoding transcription factors Ikaros and Aiolos) are susceptibility loci for systemic lupus erythematosus (SLE). The pharmacology of iberdomide (CC-220), a cereblon (CRBN) modulator targeting Ikaros and Aiolos, was studied in SLE patient cells and in a phase 1 healthy volunteer study.

Methods CRBN, IKZF1 and IKZF3 gene expression was measured in peripheral blood mononuclear cells (PBMC) from patients with SLE and healthy volunteers. Ikaros and Aiolos protein levels were measured by Western blot and flow cytometry. Anti-dsDNA and anti-phospholipid autoantibodies were measured in SLE PBMC cultures treated for 7 days with iberdomide. Fiftysix healthy volunteers were randomised to a single dose of iberdomide (0.03-6 mg, $n=6$ across seven cohorts) or placebo ( $n=2 /$ cohort). CD19+ B cells, CD3+ T cells and intracellular Aiolos were measured by flow cytometry. Interleukin (IL)-2 and IL-1 $\beta$ production was stimulated with anti-CD3 and lipopolysaccharide, respectively, in an ex vivo whole blood assay.

Results SLE patient PBMCs expressed significantly higher CRBN (1.5-fold), IKZF1 (2.1-fold) and IKZF3 (4.1fold) mRNA levels compared with healthy volunteers. Iberdomide significantly reduced Ikaros and Aiolos protein levels in B cells, T cells and monocytes. In SLE PBMC cultures, iberdomide inhibited anti-dsDNA and anti-phospholipid autoantibody production $\left(\mathrm{IC}_{50}\right.$ $\approx 10 \mathrm{nM})$. Single doses of iberdomide (0.3-6 mg) in healthy volunteers decreased intracellular Aiolos (minimum mean per cent of baseline: $\approx 12 \%-28 \%$ (B cells); $\approx 0 \%-33 \%$ (T cells)), decreased absolute CD19+ $B$ cells, increased IL-2 and decreased IL-1 $\beta$ production ex vivo.

Conclusions These findings demonstrate pharmacodynamic activity of iberdomide and support its further clinical development for the treatment of SLE. Trial registration number NCT01733875; Results.

\section{INTRODUCTION}

Ikaros and Aiolos, encoded by the IKZF1 and IKZF3 genes, respectively, are members of the Ikaros family of zinc finger transcription factors which regulate immune cell development and homeostasis. ${ }^{1}$ Polymorphisms in the IKZF1 and $I K Z F 3$ loci are associated with an increased risk of systemic lupus erythematosus (SLE). ${ }^{2-11}$ While the roles of Ikaros and Aiolos in the development of the autoimmune responses characteristic of SLE are still being studied, it is clear that Ikaros is widely expressed in haematopoietic precursors and involved in both lymphoid and myeloid cell development, with Ikaros knockout mice lacking $\mathrm{B}$ cells, and Ikaros dominant negative mice lacking $\mathrm{T}$ cells. Aiolos expression is more restricted, found in pre-B cells and mature peripheral B cells, and is required for the generation of long-lived, high-affinity plasma cells. ${ }^{1}$ Evidence suggests that Ikaros regulates key pathways such as the signal transducers and activators of transcription 4 and type 1 interferon (IFN), including the development of plasmacytoid dendritic cells, which are important producers of IFN- $\alpha .^{11}$ Notably, the IKZF1 polymorphism rs4917014 has been identified as an expression quantitative trait locus, causing the upregulation of type 1 IFN genes and the downregulation of complement genes, both of which are hallmarks of SLE pathophysiology. ${ }^{12}$ While the $I K Z F 1$ variant rs4917014*T strongly increases the 3'-UTR expression levels of IKZF1, as measured by RNA sequencing, ${ }^{12}$ the effects of the $I K Z F 3$ variants have not been clearly defined. Nonetheless, there is a compelling genetic association between these transcription factors and the immune dysfunction associated with lupus. ${ }^{2-6}$

Iberdomide (CC-220) is an orally available immunomodulatory compound under development for the treatment of SLE and relapsed/refractory multiple myeloma. This compound has been identified as a novel high-affinity ligand of cereblon $(C R B N)$, part of the cullin-ring finger ligase- 4 cereblon (CRL4 ${ }^{\mathrm{CRBN}}$ ) E3 ubiquitin ligase complex that includes cullin 4A (CUL4A), DNA damagebinding protein 1 (DDB1) and regulator of cullin 1 (ROC1). ${ }^{1314}$ Earlier studies identified cereblon as a molecular-binding target of thalidomide, lenalidomide and pomalidomide; binding of these drugs to cereblon modulates the E3 ubiquitin ligase activity of the complex, resulting in the recruitment of the zinc finger transcription factors Ikaros and Aiolos to the complex, increasing their polyubiquitination and proteasome-dependent degradation. ${ }^{14-16}$ The degradation of Ikaros and Aiolos is necessary and sufficient for inhibition of the proliferation of myeloma tumour cells, as well as for the immunomodulatory effects such as increased interleukin (IL)-2 expression by $\mathrm{T}$ cells. ${ }^{17-20}$ Knockdown of 

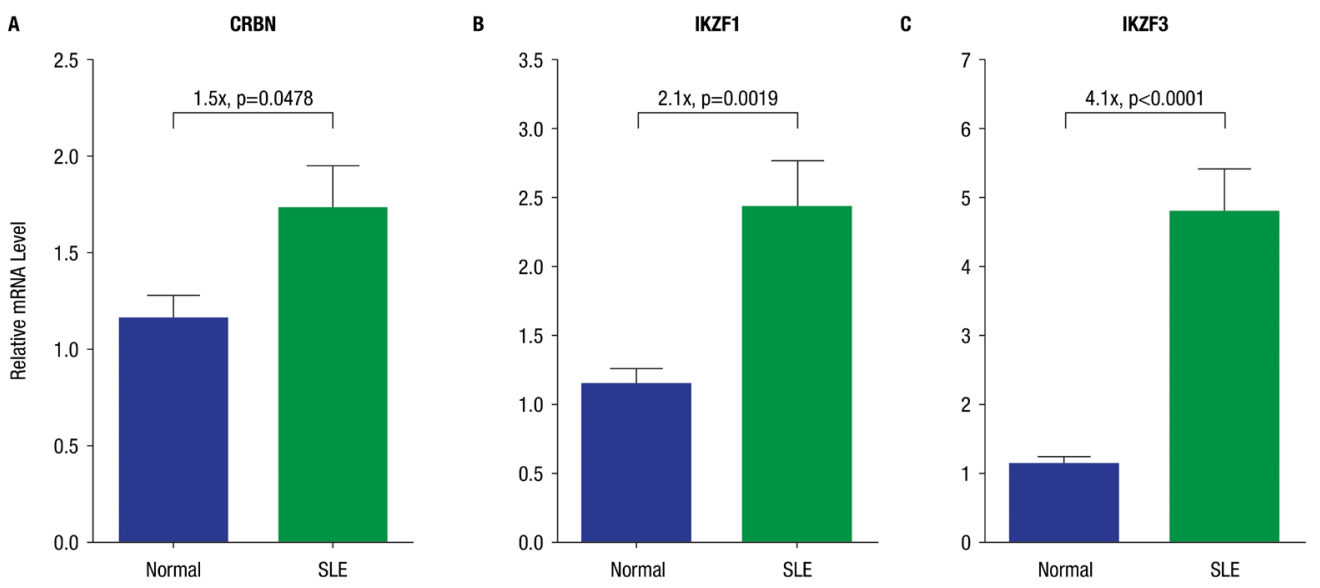

Figure 1 Overexpression of mRNA for cereblon (CRBN), Ikaros (IKZF1) and Aiolos (IKZF3) mRNA in systemic lupus erythematosus (SLE) peripheral blood mononuclear cells (PBMC). Shown are relative mRNA levels of (A) CRBN, (B) IKZF1 and (C) IKZF3 in the PBMCs of normal donors ( $\mathrm{n}=10)$ and of patients with SLE $(n=11)$. The $p$ values were generated using an unpaired t-test.

either IKZF1 or IKZF3 with shRNA in myeloma tumour cell lines markedly downregulates IRF4 expression and decreases cellular fitness. ${ }^{18}$

In previous biochemical and structural studies, it was demonstrated that CC-220 binds to cereblon with a higher affinity than lenalidomide or pomalidomide. ${ }^{13}$ This results in CC-220 having a more potent and deeper degradation of the cereblon substrates Ikaros and Aiolos. The crystal structure of CC-220 in complex with cereblon and DDB1 reveals that the increase in potency correlates with increased contacts with cereblon away from the modelled binding site for Ikaros/Aiolos. ${ }^{13}$ Thus, the structure of
CC-220 enables greater Ikaros and Aiolos degradation via tighter binding to the cereblon E3 ligase complex, enabling the use of lower doses, thereby reducing the potential for off-target effects.

In the current set of preclinical studies assessing the immunomodulatory effects of iberdomide, we explored the expression of CRBN, IKZF1 and IKZF3 in peripheral blood mononuclear cells (PBMC) from patients with SLE compared with healthy volunteers, as well as the effect of iberdomide on Ikaros and Aiolos protein levels and SLE autoantibody production in vitro. In addition, we report the impact of iberdomide on immunological parameters in a phase 1, double-blinded,
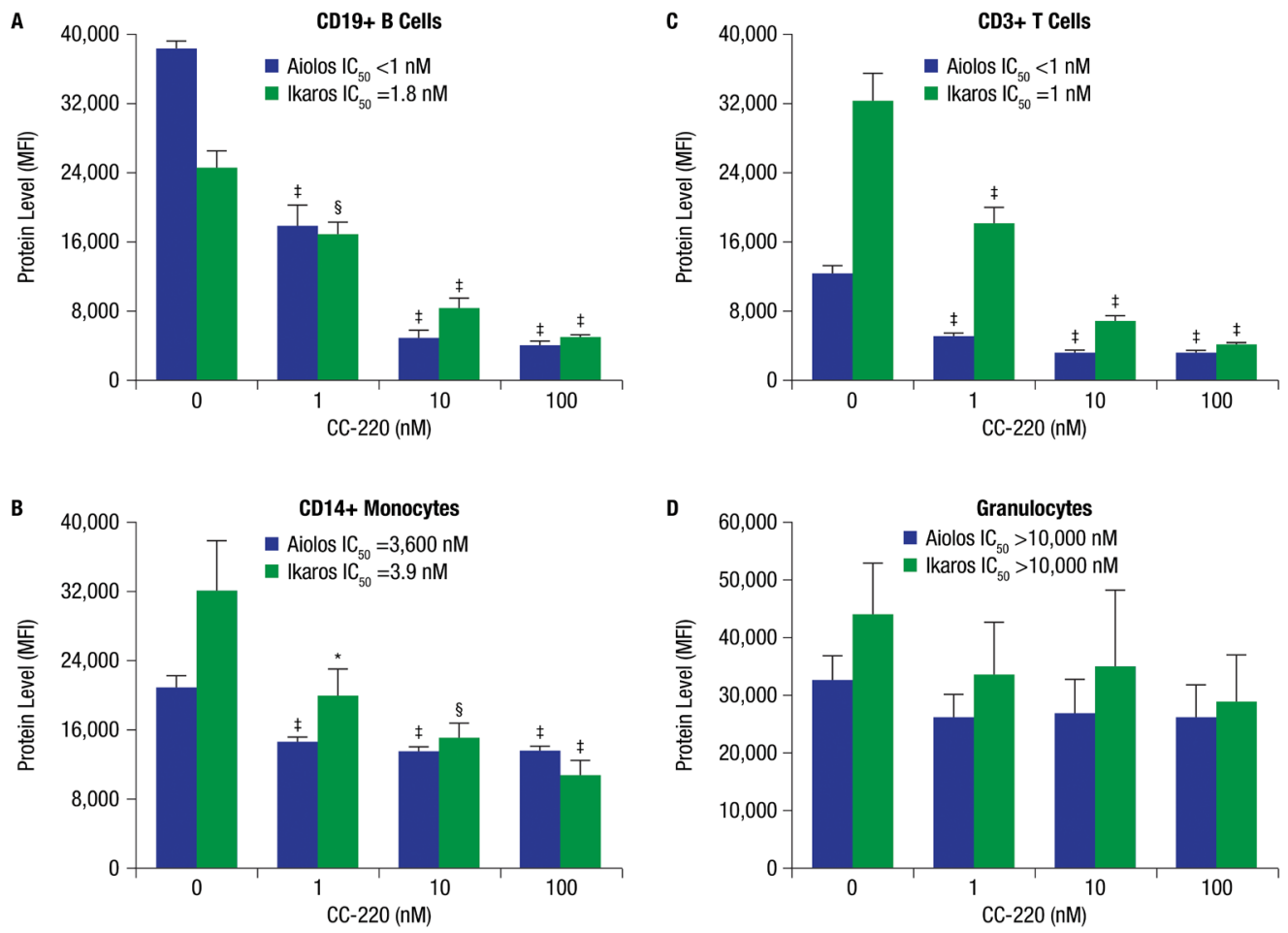

Figure 2 Iberdomide (CC-220) reduces Ikaros and Aiolos protein levels in whole blood leucocyte subsets: (A) CD19+ B cells, (B) CD14+ monocytes, (C) CD3+ T cells and (D) granulocytes. Whole blood from healthy donors was treated with CC-220 for 18 hours, lysed, fixed, permeabilised, stained with antibodies and analysed by flow cytometry. ${ }^{*} \mathrm{P} \leq 0.05 ; \S \mathrm{P} \leq 0.01 ; \neq \mathrm{P} \leq 0.001$ versus dimethyl sulfoxide (DMSO) control ( $n=3$ ), by one-way analysis of variance/Dunnett's multiple comparison test. For representative flow cytometric data, see online supplementary figures $S 1$ and $S 2$. IC ${ }_{50}$, half maximal inhibitory concentration; MFI, mean fluorescence intensity. 

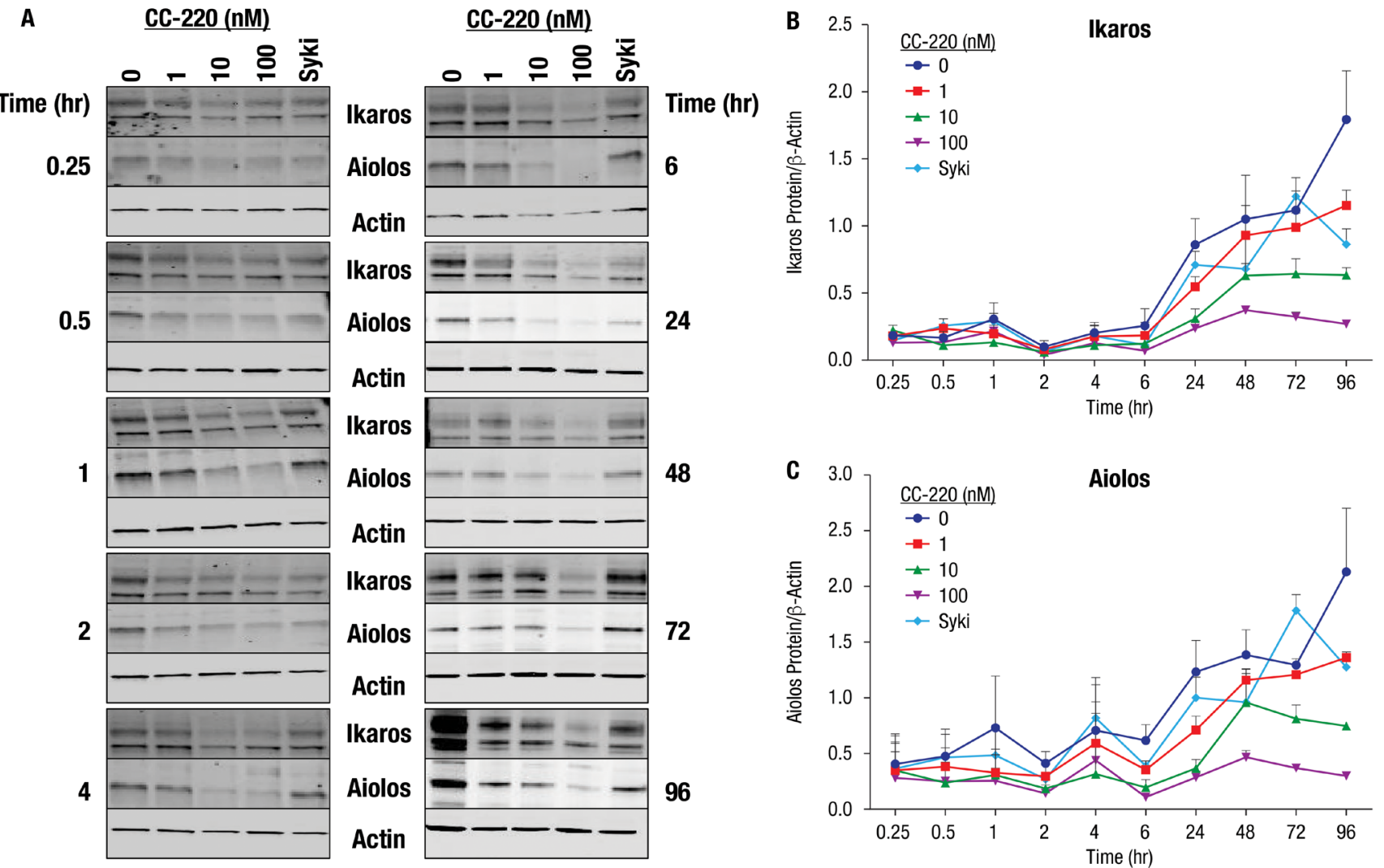

Figure 3 Effect of B cell stimulation and iberdomide (CC-220) on Ikaros and Aiolos protein levels over time. (A) CD19+ B cells were isolated from the peripheral blood mononuclear cells of normal donors and stimulated in the presence of CC-220 or a Syk inhibitor. (B, C) Ikaros and Aiolos were measured by Western blot analysis. Representative blots are shown; graphs represent the mean and SE of the mean $(n=3)$.

placebo-controlled, single-ascending dose study in healthy volunteers.

\section{METHODS}

\section{Gene expression studies}

Viably frozen PBMCs from 11 patients with SLE and 10 healthy volunteers were obtained from Conversant Bio (Huntsville, Alabama, USA). Informed consent was collected in accordance with institutional review board approval, HIPAA compliance, FDA Good Clinical Practice and all regulatory requirements (see online supplementary methods for details).

\section{Determining Ikaros and Aiolos protein levels in human whole blood by flow cytometry}

Human heparinised whole blood from normal healthy volunteers (Bioreclamation, Westbury, New York, USA) was treated with $0.1 \%$ dimethyl sulfoxide (DMSO) and iberdomide 1,10 or $100 \mathrm{nM}$ for 18 hours at $37^{\circ} \mathrm{C}, 5 \% \mathrm{CO}_{2}$. After 18 hours, the blood was lysed and fixed for flow cytometric analysis (see online supplementary methods for details).

\section{Iberdomide effect on Ikaros and Aiolos protein levels during CD19+ B cell culture}

PBMCs were generated from human buffy coats and CD19+ B cells were isolated from PBMC using the EasySep Human B Cell Enrichment Cocktail protocol (STEMCELL Technologies, Vancouver, British Columbia, Canada). The human primary B cell differentiation protocol was adapted from previously described methods. ${ }^{2122}$ See online supplementary methods for details.

\section{Iberdomide effect on SLE autoantibody production in vitro}

PBMCs were purified from heparinised whole blood from donors with SLE, treated with a B cell differentiation cocktail, as described above, and incubated with increasing concentrations of iberdomide or DMSO $(0.001 \%$ final concentration of DMSO) (see online supplementary methods for details).

\section{Phase 1 clinical trial in healthy volunteers}

In the phase 1, double-blind, placebo-controlled, single-ascending dose study, 56 healthy volunteers were randomised and enrolled in seven cohorts, with six subjects per cohort receiving a single oral dose of iberdomide $0.03-6 \mathrm{mg}$ and two subjects per cohort receiving placebo. This study was conducted in Covance Clinical Research Unit (Madison, WI, USA). Approximately $4 \mathrm{~mL}$ of blood for lymphocyte phenotyping was collected on days $-1,1$ (predose), 2 (24 hours postdose) and 3 (48 hours postdose) and at the follow-up visit (day 5). Blood was also collected predose and at 3,12 and 24 hours postdose for ex vivo cytokine production assessment and transcription factor quantification. For cohorts administered the 4 and $6 \mathrm{mg}$ doses of iberdomide, additional blood samples were taken on days 5 and 7, and at the follow-up visit (day 9). CD19+ B cells, CD3+ T cells and intracellular Aiolos were measured by flow cytometry. IL-2 and IL-1 $\beta$ production was stimulated with anti-CD3 and lipopolysaccharide (LPS), respectively, in the TruCulture ex vivo 
whole blood assay system (Myriad RBM, Austin, Texas). Results were reported as per cent of baseline at each time point. The SAS Enterprise Guide V.4.1 software was used for data analysis (SAS Institute). All statistical tests were conducted with a two-sided significance level of 0.05 . For parameters before and after iberdomide treatment, a Wilcoxon signed-rank test was used to compare raw values and per cent of baseline at each postbaseline time point with baseline values.

\section{RESULTS}

Compared with PBMC from normal donors, PBMCs from patients with SLE expressed significantly higher levels of mRNA for CRBN (1.5-fold), IKZF1 (2.1-fold) and IKZF3) (4.1-fold) (figure $1 \mathrm{~A}-\mathrm{C}$ ). Treatment of whole blood from healthy donors with increasing concentrations of iberdomide $1-100 \mathrm{nM}$ significantly reduced Ikaros and Aiolos protein levels in B cells, T cells and monocytes, but not granulocytes (figure 2A-D and figure $3 \mathrm{~A}-\mathrm{C}$ ). Reductions in Ikaros and Aiolos protein levels were significant versus control at the $1 \mathrm{nM}$ concentration of iberdomide in B cells, T cells and monocytes (figure 2A-C and online supplementary figures S1 and S2); the $\mathrm{IC}_{50}$ for Aiolos was $<1 \mathrm{nM}$ in $\mathrm{B}$ cells and $\mathrm{T}$ cells, and the $\mathrm{IC}_{50}$ for Ikaros was $1.8 \mathrm{nM}$ in B cells and $1 \mathrm{nM}$ in T cells.

To address the specificity of the iberdomide-induced cereblon substrate degradation, other proteins with homology to Ikaros and Aiolos were measured in B cells and $\mathrm{T}$ cells using microcapillary electrophoresis western blotting. Treatment of B cells with iberdomide $(10 \mathrm{nM}$ for 24 hours) resulted in a significant decrease in the protein levels of Ikaros $(66 \mathrm{kDa}$ isoform) and Aiolos $(58 \mathrm{kDa}$ isoform), but not the other Ikaros family zinc finger factors Helios (IKZF2) or Pegasus (IKZF5) (see online supplementary figure S3). Expression of Eos (IKZF4) was not detected in B cells. Similarly, treatment of T cells with iberdomide ( $10 \mathrm{nM}$ for 24 hours) resulted in a significant decrease in the protein levels of Ikaros (58 and $66 \mathrm{kDa}$ isoforms) and Aiolos (58 and $80 \mathrm{kDa}$ isoforms), but not Helios, Eos or Pegasus. Another zinc finger protein with sequence homology to Ikaros and Aiolos, Zinc Finger Protein 91 (ZFP91), was significantly reduced by iberdomide ( $10 \mathrm{nM}$ for 24 hours) in B cells (see online supplementary figure S3).

In stimulated B cells, Ikaros and Aiolos protein levels were reduced by iberdomide treatment as early as 0.25 hour after treatment initiation (figure 3A). Compared with protein expression levels at time zero (cell culture initiation), Ikaros and Aiolos protein levels rose noticeably after 24-96 hours in the culture as a result of $\mathrm{B}$ cell activation. Under these B cell-activating conditions, iberdomide suppressed the Ikaros and Aiolos protein expression in a concentration-dependent manner (figure 3B,C).

In cultures of PBMCs from patients with SLE $(n=10)$, iberdomide inhibited anti-dsDNA and anti-phospholipid IgM autoantibody production with an $\mathrm{IC}_{50}$ of approximately $10 \mathrm{nM}$ in each case (figure 4A,B). Greater than 50\% inhibition of anti-dsDNA and anti-phospholipid autoantibody production was observed in most donors at the $100 \mathrm{nM}$ concentration of iberdomide, and at least $40 \%-50 \%$ inhibition at the $10 \mathrm{nM}$ concentration in five of the 10 donors (see online supplementary table S1).

The pharmacodynamics of iberdomide were studied in a single-ascending dose phase 1 trial in healthy volunteers. In these subjects, a treatment-related decrease from baseline in intracellular Aiolos levels was observed following administration of iberdomide at $0.3-6 \mathrm{mg}$ doses, with a mean per cent of baseline of $24.8 \%$ in B cells and $0 \%$ in T cells at 24 hours after administration of the $0.3 \mathrm{mg}$ dose (figure $5 \mathrm{~A}, \mathrm{~B}$ ). In B cells at 12 hours postdose, Aiolos levels were $28.2 \%$ of baseline in the iberdomide
A

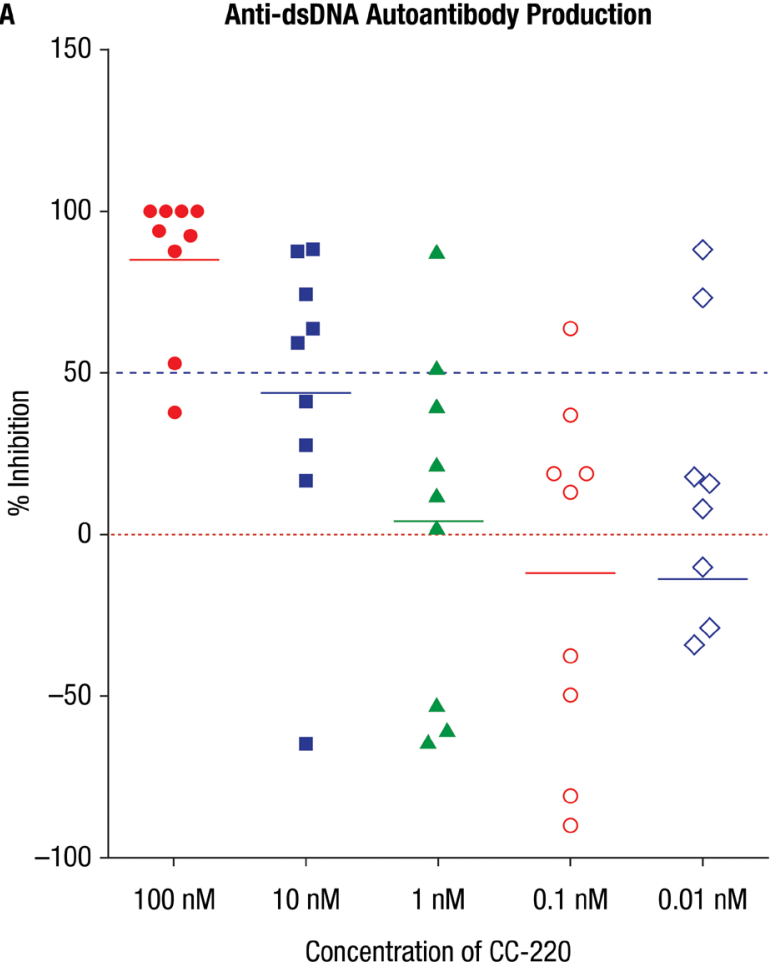

B

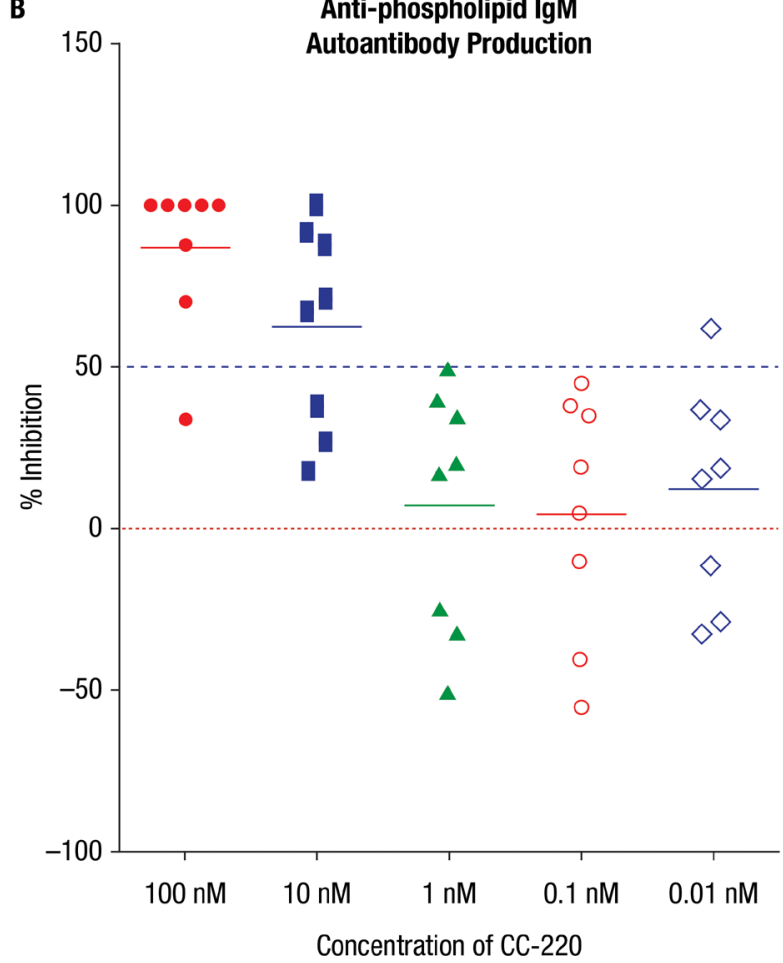

Figure 4 Iberdomide (CC-220) inhibits (A) anti-dsDNA and (B) anti-phospholipid IgM (systemic lupus erythematosus) autoantibody production in vitro. In cultures of peripheral blood mononuclear cells from patients with systemic lupus erythematosus, CC-220 inhibited production of anti-dsDNA autoantibodies $(n=9)$ and anti-phospholipid autoantibodies $(n=8)$ with a half maximal inhibitory concentration of approximately $10 \mathrm{nM}$. 
A

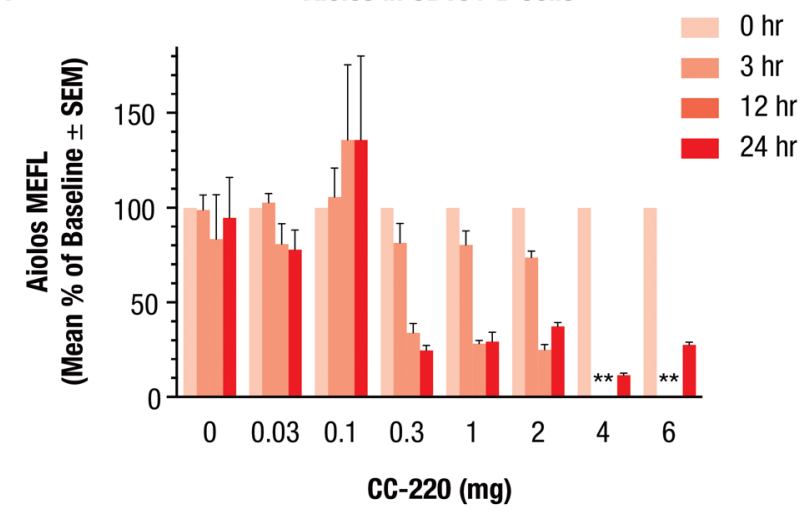

B

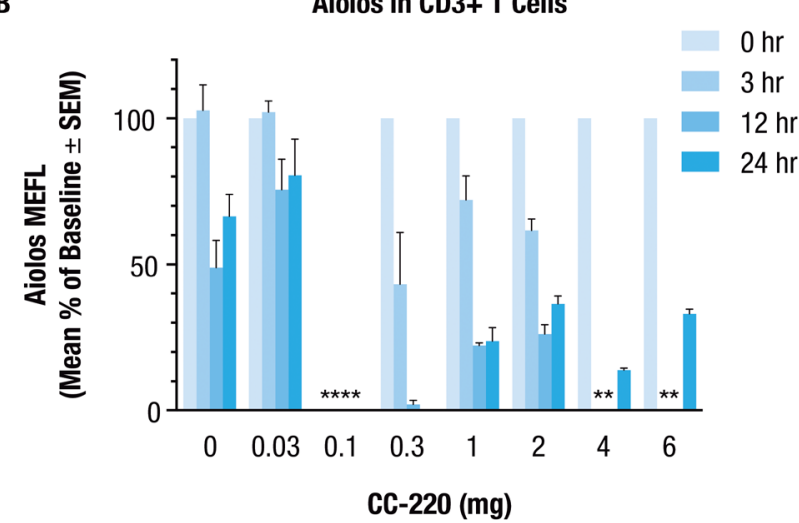

C

Absolute CD19+ B-Cell Counts

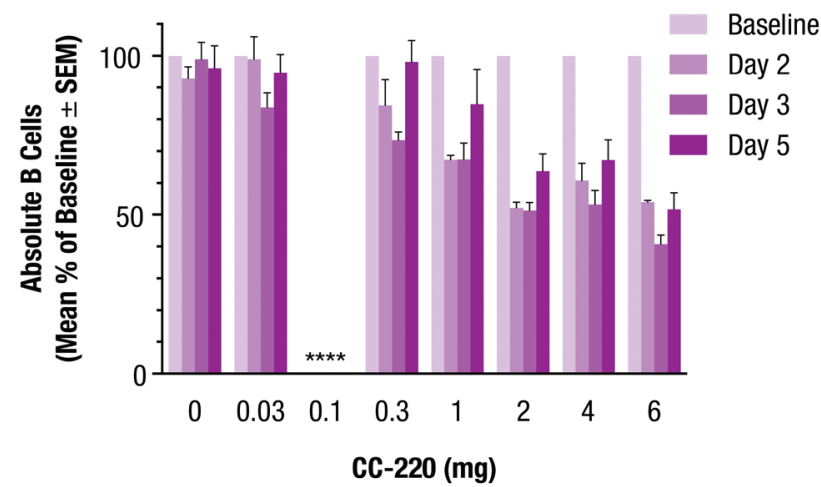

D

Absolute CD3+ T-Cell Counts

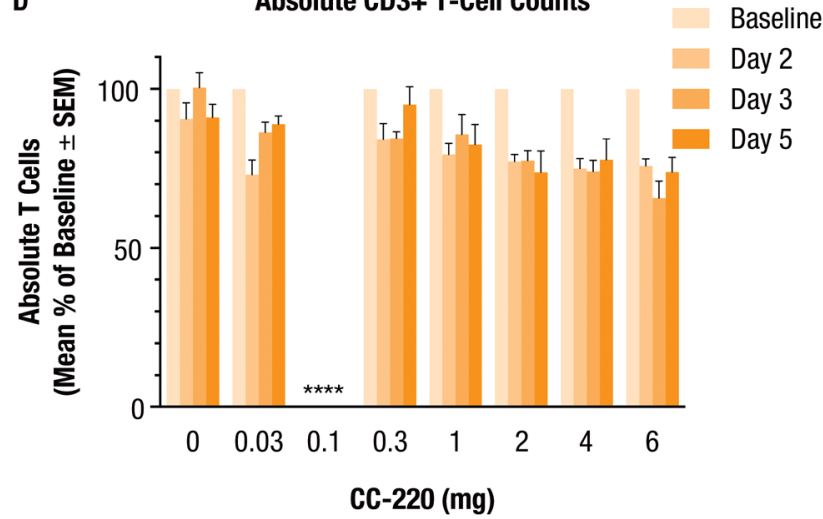

Figure 5 Effect of a single dose of iberdomide (CC-220) on Aiolos expression in (A) CD19+ B cells and (B) CD3+ T cells, and effect on (C) peripheral blood CD19+ B cell counts and (D) CD3+ T cell counts in healthy volunteers. Subjects were randomised to single doses of placebo (0) or CC-220 $0.03 \mathrm{mg}$ (cohort A), $0.1 \mathrm{mg}$ (cohort B), $0.3 \mathrm{mg}$ (cohort C), $1 \mathrm{mg}$ (cohort D) or $2 \mathrm{mg}$ (cohort E). Aiolos expression was measured as per cent of baseline at 3,12 and 24 hours postdose. Peripheral blood B cell and T cell counts were measured as per cent of baseline at day 2, day 3 and day 5 postdose. $\mathrm{n}=6$ per CC-220 treatment group, $\mathrm{n}=14$ for pooled placebo groups. *Data not available. (Due to inclement weather at the clinical site during the dosing of the $0.1 \mathrm{mg}$ cohort, some flow cytometric samples were not received for analysis within the stability window.) MEFL, molecules equivalent fluorescence.

$1 \mathrm{mg}$ group and $25.0 \%$ of baseline in the iberdomide $2 \mathrm{mg}$ group. In B cells at 24 hours postdose, Aiolos levels were $11.5 \%$ of baseline in the iberdomide $4 \mathrm{mg}$ group and $27.7 \%$ of baseline in the iberdomide $6 \mathrm{mg}$ group (data not shown). In T cells at 12 hours postdose, Aiolos levels were $22.3 \%$ of baseline in the iberdomide $1 \mathrm{mg}$ group and $26.1 \%$ of baseline in the iberdomide $2 \mathrm{mg}$ group. In T cells at 24 hours postdose, Aiolos levels were $13.8 \%$ of baseline in the iberdomide $4 \mathrm{mg}$ group and $33.1 \%$ of baseline in the iberdomide $6 \mathrm{mg}$ group (data not shown). In B cells and T cells, the mean per cent of baseline values compared with baseline was statistically significant at 3 hours postdose for the iberdomide $2 \mathrm{mg}$ group, at 12 hours postdose for the iberdomide $0.3 \mathrm{mg}$ group and at 24 hours postdose for the iberdomide 0.3 and $2 \mathrm{mg}$ groups (all $\mathrm{p}<0.05$ ).

An iberdomide treatment-related decrease was noted in absolute CD19+ Bcell counts, and a more modest effect on CD3 + Tcell counts in healthy volunteers for doses between 0.3 and $6 \mathrm{mg}$, with minimum mean per cent of baseline values of $73.5 \%$ for B cells on day 3, and $84.1 \%$ for T cells on day 2 after administration of the $0.3 \mathrm{mg}$ dose (figure 5C,D). For absolute CD19+ B cell counts, the effect of iberdomide appeared to be dose related: the mean per cent of baseline values was $67.3 \%$ on day 2 in the iberdomide $1 \mathrm{mg}$ group $(\mathrm{p}<0.05)$ and $51.3 \%$ on day 3 in the iberdomide $2 \mathrm{mg}$ group $(\mathrm{p}<0.05)$; on day 3 , the mean per cent of baseline values was $53.3 \%$ in the iberdomide $4 \mathrm{mg}$ group and $40.8 \%$ in the iberdomide $6 \mathrm{mg}$ group $(\mathrm{p}<0.05)$. For absolute CD $3+$ T cell counts, the mean per cent of baseline was $79.4 \%$ on day 2 in the iberdomide $1 \mathrm{mg}$ group (not significant), and $73.8 \%$ on day 5 in the iberdomide 2 mg group $(\mathrm{p}<0.05)$; on day 3 , the mean per cent of baseline was $74.0 \%$ in the iberdomide $4 \mathrm{mg}$ group and $65.7 \%$ in the iberdomide $6 \mathrm{mg}$ group (both $\mathrm{p}<0.05$ ).

Furthermore, iberdomide administration resulted in increased IL-2 production and decreased IL- $1 \beta$ production in whole blood ex vivo (figure $6 \mathrm{~A}, \mathrm{~B}$ ). For anti-CD3-stimulated IL-2 production, the mean per cent of baseline was $315 \%, 973 \%$ and $915 \%$ at 12 hours after administration of the iberdomide $0.3,1$ and $2 \mathrm{mg}$ doses (all not significant), respectively (figure 6A), and $779 \%$ and $1896 \%$ at 24 hours for the iberdomide 4 and $6 \mathrm{mg}$ groups, respectively (both not significant). Following anti-CD3 stimulation, there was also increased production of IFN- $\gamma$ from 1 to $6 \mathrm{mg}$ iberdomide, with a maximum mean per cent of baseline of approximately $304 \%$ for IFN- $\gamma$ after administration of $1 \mathrm{mg}$ iberdomide. There was also increased production of the cytokine granulocyte-macrophage colony-stimulating factor at the highest dose levels (4 and $6 \mathrm{mg}$ ). For LPS-stimulated IL-1 $\beta$ production, the mean per cent of baseline was $49.5 \%(p<0.05), 21.8 \%$ (not significant) and $16.3 \%(\mathrm{p}<0.05)$ at 12 hours after administration 
A

Anti-CD3-Induced IL-2

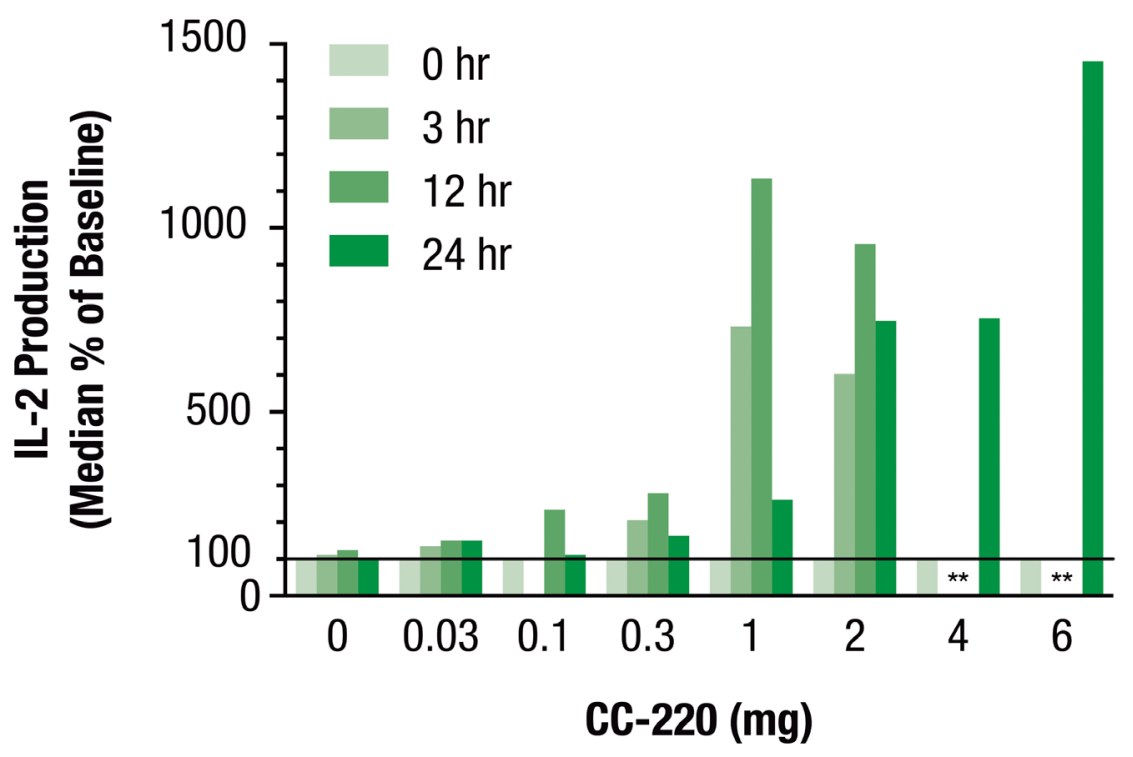

B LPS-Induced IL-1 $\beta$

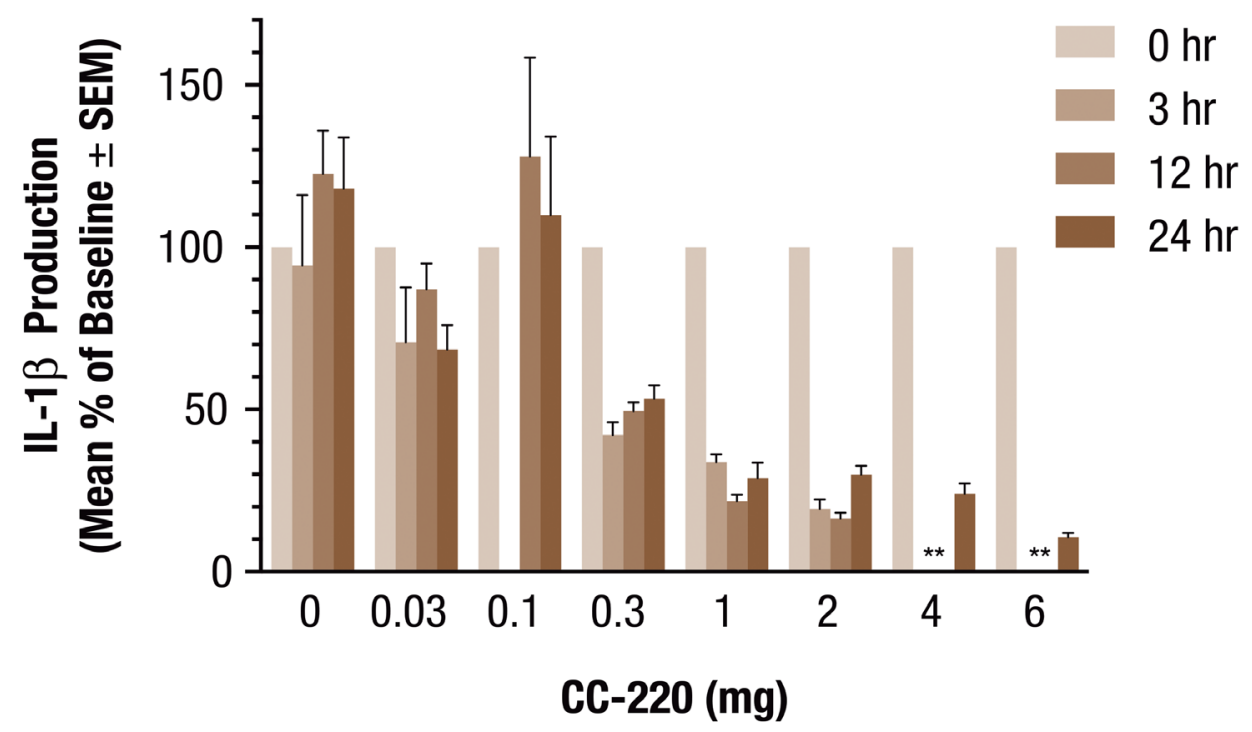

Figure 6 A single dose of iberdomide (CC-220) increased interleukin (IL)-2 production in (A) anti-CD3-stimulated whole blood and (B) decreases IL$1 \beta$ production by lipopolysaccharide (LPS)-stimulated whole blood ex vivo in healthy volunteers. Subjects were randomised to single doses of placebo (0) or CC-220 $0.03 \mathrm{mg}$ (cohort A), $0.1 \mathrm{mg}$ (cohort B), $0.3 \mathrm{mg}$ (cohort C), $1 \mathrm{mg}$ (cohort D) or $2 \mathrm{mg}$ (cohort E). IL-2 (pg/mL) and IL-1 $1 \beta \mathrm{production} \mathrm{by} \mathrm{ex}$ vivo anti-CD3 or LPS-stimulated whole blood, respectively, was measured as per cent of baseline at 3, 12 and 24 hours postdose. $n=6$ per $C C-220$ treatment group, $\mathrm{n}=10$ for pooled placebo groups. *Data not available.

of the iberdomide $0.3,1$ and $2 \mathrm{mg}$ doses, respectively (figure $6 \mathrm{~B}$ ), and $24.1 \%(\mathrm{p}<0.05)$ and $10.7 \%$ (not significant) at 24 hours for the iberdomide 4 and $6 \mathrm{mg}$ doses, respectively. Following LPS stimulation, other proinflammatory cytokines IL-1 $\alpha$, IL- 6 and tumour necrosis factor (TNF)- $\alpha$ were also inhibited in a generally dose-related manner across the $0.3-6 \mathrm{mg}$ iberdomide dose range. The minimum pharmacologically active dose, $0.3 \mathrm{mg}$ iberdomide, had minimum mean per cent of baseline values of approximately $40 \%, 43 \%$ and $44 \%$ for IL- $1 \alpha$, IL- 6 and TNF- $\alpha$, respectively.

In this single-ascending dose study in healthy volunteers, there were no serious adverse events or severe treatment-emergent adverse events (TEAE) reported, and no subjects withdrew from the study due to a TEAE. All TEAEs were mild in severity with the exception of one case of moderate urticaria in the $4 \mathrm{mg}$ dose group. There were no clinically significant or 
apparent drug or dose-related trend changes or findings noted in clinical laboratory evaluations, vital sign measurements, physical examinations or neurological examinations for this study. Overall, there were no remarkable clinical safety findings during the study. Iberdomide was well tolerated when administered as single oral doses up to $6 \mathrm{mg}$ in this group of healthy subjects.

\section{DISCUSSION}

Iberdomide (CC-220) is a novel oral immunomodulatory compound that targets cereblon, part of the CRL4 ${ }^{\mathrm{CRBN}} \mathrm{E} 3$ ubiquitin ligase complex. We and others have shown that binding of thalidomide and its analogues to cereblon results in recruitment and increased degradation of the transcription factors Ikaros and Aiolos. Furthermore, genetic studies have associated polymorphisms in the genes encoding Ikaros (IKZF1) and Aiolos (IKZF3) with the risk of developing SLE, and these transcription factors may play a role in the pathogenesis of the disease. The first set of in vitro experiments reported here was conducted to assess expression levels of these genes of interest in patients with SLE, and indeed confirmed that CRBN, IKZF1 and IKZF3 mRNA are overexpressed in the PBMCs of patients with SLE relative to healthy controls.

Further in vitro experiments were conducted to more clearly define the immunomodulatory effects of iberdomide. These experiments demonstrated that the addition of iberdomide at concentrations as low as $1 \mathrm{nM}$ resulted in a potent reduction in Ikaros and Aiolos protein levels in B cells, $\mathrm{T}$ cells and monocytes from healthy donors. The other Ikaros family zinc finger transcription factors, namely Helios, Eos and Pegasus, were not significantly reduced by treatment of B cells or T cells with iberdomide, illustrating that there is selectivity for cereblon substrates even within this group of related proteins. However, another zinc finger protein with sequence homology to Ikaros and Aiolos, ZFP91, was also degraded in iberdomide-treated B cells, demonstrating that the effects of iberdomide on other cereblon substrates cannot be ruled out.

The reduction in Ikaros and Aiolos in B cells was associated with inhibition of anti-dsDNA and anti-phospholipid IgM autoantibody production in the PBMCs of patients with SLE with an $\mathrm{IC}_{50}$ of approximately $10 \mathrm{nM}$. This inhibitory effect on immunoglobulin production is not specific to autoantibodies, since iberdomide inhibited $\mathrm{B}$ cell differentiation to plasmablast differentiation, and production of $\operatorname{IgG}$ and $\operatorname{IgM}$, to a similar extent whether the B cells were isolated from patients with SLE or from healthy donors. ${ }^{23}$

Iberdomide effects on the immune system were also confirmed in pharmacodynamic results from a phase 1, placebo-controlled, single-ascending dose study in healthy volunteers. In this study, administration of single doses of iberdomide $0.3-6 \mathrm{mg}$ reduced intracellular Aiolos protein expression in B cells and $\mathrm{T}$ cells, and reduced absolute $\mathrm{B}$ cell counts, and to a lesser degree $\mathrm{T}$ cell counts in the peripheral blood. Iberdomide administration also increased T cell-derived IL-2 production and decreased LPS-induced IL-1 $\beta$ production in whole blood ex vivo. The pharmacologic effects of iberdomide are therefore at least tripartite in nature: (1) reduction of absolute B cell counts; (2) augmentation of IL-2 production by T cells; and (3) inhibition of IL- $1 \beta$ production in response to a proinflammatory stimulus. This constellation of effects provides a unique opportunity for the treatment of autoimmune conditions such as SLE, which are characterised by autoantibody production, deficiencies in regulatory $\mathrm{T}$ cells and a proinflammatory cytokine milieu.
Taken together, these findings support the further development of iberdomide for the treatment of SLE and other autoimmune diseases. A pilot, phase 2a, randomised, double-blind, placebo-controlled study of iberdomide is ongoing (ClinicalTrials.gov Identifier: NCT02185040) ${ }^{24}$ to evaluate its effectiveness in patients with active SLE.

Acknowledgements The authors received editorial support in the preparation of this report from Peloton Advantage, funded by Celgene. The authors, however, directed and are fully responsible for all content and editorial decisions for this manuscript. During manuscript review, iberdomide was adopted as the recommended International Nonproprietary Name (INN) for CC-220. For future clarity, inclusion of the INN name was therefore made throughout the manuscript.

Contributors PHS, YY, LW, JK, GR, MT, MP and RC conceptualised and designed the study. LW and JK conducted the preclinical experiments. PHS, YY, MT, MP and RC planned and conducted the clinical trial. All authors were integral in the interpretation of the results. ZY and LL performed the programming and statistical analysis of the clinical data. PHS, YY, LW, JK and GR prepared the data and the first draft of the manuscript. All authors were involved in critical review of the data as well as drafting and revising the manuscript, and all have approved the final version of the paper to be published.

Funding This study was sponsored by Celgene, Summit, New Jersey, USA.

Competing interests The authors either are, or have been, employees and shareholders of Celgene.

Patient consent Not required.

Ethics approval Schulman Associates IRB

Provenance and peer review Not commissioned; externally peer reviewed.

Open access This is an open access article distributed in accordance with the Creative Commons Attribution Non Commercial (CC BY-NC 4.0) license, which permits others to distribute, remix, adapt, build upon this work non-commercially, and license their derivative works on different terms, provided the original work is properly cited and the use is non-commercial. See: http://creativecommons.org/ licenses/by-nc/4.0/

(c) Article author(s) (or their employer(s) unless otherwise stated in the text of the article) 2018. All rights reserved. No commercial use is permitted unless otherwise expressly granted.

\section{REFERENCES}

1 John LB, Ward AC. The Ikaros gene family: transcriptional regulators of hematopoiesis and immunity. Mol Immunol 2011;48:1272-8.

2 Cunninghame Graham DS, Morris DL, Bhangale TR, et al. Association of NCF2, IKZF1, IRF8, IFIH1, and TYK2 with systemic lupus erythematosus. PLoS Genet 2011:7:e1002341.

3 Lessard CJ, Adrianto I, Ice JA, et al. Identification of IRF8, TMEM39A, and IKZF3ZPBP2 as susceptibility loci for systemic lupus erythematosus in a large-scale multiracial replication study. Am J Hum Genet 2012;90:648-60.

4 Han JW, Zheng HF, Cui Y, et al. Genome-wide association study in a Chinese Han population identifies nine new susceptibility loci for systemic lupus erythematosus. Nat Genet 2009;41:1234-7.

5 He CF, Liu YS, Cheng YL, et al. TNIP1, SLC15A4, ETS1, RasGRP3 and IKZF1 are associated with clinical features of systemic lupus erythematosus in a Chinese Han population. Lupus 2010;19:1181-6.

6 Leng RX, Wang W, Cen H, et al. Gene-gene and gene-sex epistatic interactions of MiR146a, IRF5, IKZF1, ETS1 and IL21 in systemic lupus erythematosus. PLoS One 2012; 7:e51090.

7 Wang C, Ahlford A, Järvinen TM, et al. Genes identified in Asian SLE GWASs are also associated with SLE in Caucasian populations. Eur J Hum Genet 2013;21:994-9.

8 Cai X, Qiao Y, Diao C, et al. Association between polymorphisms of the IKZF3 gene and systemic lupus erythematosus in a Chinese Han population. PLoS One 2014;9:e108661

9 You Y, Zhai ZF, Chen FR, et al. Autoimmune risk loci of IL12RB2, IKZF1, XKR6, TMEM39A and CSK in Chinese patients with systemic lupus erythematosus. Tissue Antigens 2015;85:200-3.

10 Dang J, Shan S, Li J, et al. Gene-gene interactions of IRF5, STAT4, IKZF1 and ETS1 in systemic lupus erythematosus. Tissue Antigens 2014;83:401-8.

11 Hu SJ, Wen LL, Hu X, et al. IKZF1: a critical role in the pathogenesis of systemic lupus erythematosus? Mod Rheumatol 2013;23:205-9.

12 Westra HJ, Peters MJ, Esko T, et al. Systematic identification of trans eQTLs as putative drivers of known disease associations. Nat Genet 2013:45:1238-43.

13 Matyskiela ME, Zhang W, Man HW, et al. A cereblon modulator (CC-220) with Improved Degradation of Ikaros and Aiolos. J Med Chem 2018;61:535-42.

14 Ito T, Ando H, Suzuki T, et al. Identification of a primary target of thalidomide teratogenicity. Science 2010;327:1345-50. 
15 Lopez-Girona A, Mendy D, Ito T, et al. Cereblon is a direct protein target for immunomodulatory and antiproliferative activities of lenalidomide and pomalidomide. Leukemia 2012;26:2326-35.

16 Zhu YX, Kortuem KM, Stewart AK. Molecular mechanism of action of immunemodulatory drugs thalidomide, lenalidomide and pomalidomide in multiple myeloma. Leuk Lymphoma 2013;54:683-7.

17 Gandhi AK, Kang J, Havens CG, et al. Immunomodulatory agents lenalidomide and pomalidomide co-stimulate T cells by inducing degradation of T cell repressors Ikaros and Aiolos via modulation of the E3 ubiquitin ligase complex CRL4(CRBN.). Br J Haematol 2014;164:811-21.

18 Lu G, Middleton RE, Sun H, et al. The myeloma drug lenalidomide promotes the cereblon-dependent destruction of Ikaros proteins. Science 2014;343:305-9.

19 Chamberlain PP, Lopez-Girona A, Miller K, et al. Structure of the human CereblonDDB1-lenalidomide complex reveals basis for responsiveness to thalidomide analogs. Nat Struct Mol Biol 2014;21:803-9.
20 Bjorklund CC, Lu L, Kang J, et al. Rate of CRL4(CRBN) substrate Ikaros and Aiolos degradation underlies differential activity of lenalidomide and pomalidomide in multiple myeloma cells by regulation of c-Myc and IRF4. Blood Cancer J 2015;5:e354.

21 Jourdan M, Caraux A, De Vos J, et al. An in vitro model of differentiation of memory B cells into plasmablasts and plasma cells including detailed phenotypic and molecular characterization. Blood 2009;114:5173-81.

22 Bellatin MF, Han M, Fallena M, et al. Production of autoantibodies against citrullinated antigens/peptides by human B cells. J Immuno/ 2012;188:3542-50.

23 Nakayama Y, Kosek J, Capone L, et al. Aiolos overexpression in systemic lupus erythematosus B cell subtypes and BAFF-induced memory B cell differentiation are reduced by CC-220 modulation of cereblon activity. J Immunol 2017;199:2388-407.

24 Schafer $P, Y e$ Y, Wu L, et al. CC-220, a novel immunomodulatory compound, in a pilot, phase 2, randomized, placebo-controlled, double-blind study to evaluate efficacy, safety, tolerability, pharmacokinetics, pharmacodynamics, and pharmacogenetics of CC-220 in subjects with systemic lupus erythematosus [poster]. Proceeding of the Triennial International Congress on SLE; September 18-20, Quebec City, QC, Canada, 2014 